Published in "Computational Materials Science (2): 263-270, 2006"

which should be cited to refer to this work.

\title{
Ab initio study of structural, electronic, elastic and high pressure properties of barium chalcogenides
}

\author{
A. Bouhemadou ${ }^{\mathrm{a}, *}$, R. Khenata ${ }^{\mathrm{b}}$, F. Zegrar ${ }^{\mathrm{a}}$, M. Sahnoun ${ }^{\mathrm{c}}$, H. Baltache ${ }^{\mathrm{b}}$, A.H. Reshak ${ }^{\mathrm{d}}$ \\ a Département de Physique, Faculté des Sciences, Université Ferhat Abbes, 19000 Sétif, Algeria \\ b Laboratoire de Physique Quantique et de Modélisation Mathématique (LPQ3M), Département de Technologie, \\ Université de Mascara, 29000 Mascara, Algeria \\ ${ }^{\mathrm{c}}$ Département de Chimie, Université de Fribourg, Fribourg Ch-1700, Switzerland \\ d Physics Department, Indian Institute of Technology, 247667 Roorkee, Uttaranchal, India
}

\begin{abstract}
A theoretical studies of structural, electronic, elastic and high pressure properties in barium chalcogenides $\mathrm{BaX}(\mathrm{X}=\mathrm{S}, \mathrm{Se}, \mathrm{Te}, \mathrm{Po})$ are performed, using the full-potential augmented plane wave plus local orbitals method (FP-APW + lo). In this approach the local-density approximation (LDA) and generalized gradient approximation (GGA) are used for the exchange-correlation (XC) potential. Moreover, the alternative form of GGA proposed by Engel and Vosko (GGA-EV) is also used for band structure calculations. The equilibrium lattice constant and the bulk modulus agree well with the experiments. The pressures at which these compounds undergo structural phase transition from $\mathrm{NaCl}$ (B1) to $\mathrm{CsCl}$ (B2) phase were found to be in good agreement with the available experimental data. Results obtained for band structure using GGA-EV show a significant improvement over other theoretical work and are closer to the experimental data. A linear relationship is observed between theoretical band gap and $1 / a^{2}$ (where $a$ is lattice constant). We have determined the elastic constants $C_{11}, C_{12}$ and $C_{44}$ at ambient conditions in both B1 and B2 structures, which have not been established neither experimentally nor theoretically. Further, we have also calculated the pressure dependence of the elastic constants for the B1 structure of the four compounds.
\end{abstract}

PACS: 71.15.Mb; 71.15.Ap; 71.15.La; 61.50.Ks

Keywords: Semiconductors; Barium chalcogenides; FP-LAPW; GGA-EV; Phase transition; Elastic constants; Electronic structure

\section{Introduction}

Alkaline-earth chalcogenide compounds are currently under intense investigations driven by their potential application in light-emitting diodes (LEDs) and laser diodes (LDs). It is expected that these compounds may provide new II-VI candidates for the fabrication of various electrical and optical devices [1]. Among the wide-band-gap II-VI semiconductors, the barium chalcogenides BaX

\footnotetext{
* Corresponding author. Tel.: +21336925128; fax: +21336925101.

E-mail address: a_bouhemadou@yahoo.fr (A. Bouhemadou).
}

$(\mathrm{X}=\mathrm{S}, \mathrm{Se}, \mathrm{Te}, \mathrm{Po})$ and their alloys, are interesting in connection with optoelectronic applications in the bluelight wavelength regime, and an understanding of their electronic and optical properties is therefore important. These may be tuned by means of diverse bond characteristics [2-5], and by means of built-in strains produced by epitaxial growth of heterostructures under controlled conditions. Prediction and analysis of such effects require knowledge of the elastic constants and relevant deformation potentials of the constituents. Under pressure $\mathrm{BaS}$, $\mathrm{BaSe}$ and $\mathrm{BaTe}$ compounds undergo a structural phase transition from the six fold-coordinated B1 structure to 
the eight fold-coordinated CsCl-type (B2) structure at the respective pressure $6.5,6.0$ and $4.8 \mathrm{GPa}$, as it has been shown by high pressure X-ray diffraction experiments [6-9].

From a theoretical point of view, several first-principle calculations were made for $\mathrm{BaS}, \mathrm{BaSe}$ and $\mathrm{BaTe}$ compounds by a variety of methods [5,10-16]. Wei and Krakauer [10] have used the linearized augmented plane wave (LAPW) method to study both structural and metallization transition of BaSe and BaTe. The augmented-spherical wave method within the local-density approximation (LDA-ASW) has been used by Carlsson and Wilkins [11] to discuss the metallization transition volume of BaS, $\mathrm{BaSe}$ and BaTe. Kalpana and co-workers [12] have studied the electronic structure and structural phase stability of these compounds by means of the tight-binding linear muffin-orbital (TB-LMTO) method. The high pressure structural phase transition of BaSe and BaTe have also been investigated by Jha et al. [13] using the three body potential approach. Syassen and co-workers [14], using the linear muffin-tin-orbital (LMTO) method, Akbarzadeh et al. [15], using the full-potential linear augmented plane wave (FP-LAPW) and the pseudo-potential plane wave (PP-PW) methods, reported some band structures of BaTe compound. In more recent study Lin and co-workers [5] have used the pseudo-potential plane wave to study the relationship between electronic properties and chemical bonds in BaX compounds. Khenata et al. [16] and Pouraghazi et al. [17] reported some band structure results on these compounds. The authors [16,17] used the fullpotential linearized augmented plane wave (FP-LAPW) method.

Despite these studies, some interesting problems remain unresolved and a number of basis properties are still unknown. To the best of our knowledge no experimental or theoretical studies on the elastic constants in both $\mathrm{NaCl}$ and $\mathrm{CsCl}$ structures of $\mathrm{BaS}, \mathrm{BaSe}, \mathrm{BaTe}$ and $\mathrm{BaPo}$ have been carried out. No experimental or theoretical studies appeared in the literature on the high pressure properties of BaPo compound. No ab initio calculations within the Engel-Vosko scheme for the exchange correlation potential were performed for any of the BaX compounds. When theoretical calculations are available, there are always lacks of information about quantities in which we are interested. This lacks of information has motivated us to explore the structural, elastic and electronic properties of $\mathrm{BaX}$ compounds. Therefore, the aim of this paper is to provide a comparative study of structural and electronic properties and a prediction study of the elastic properties under high pressure by using the full-potential augmented plane wave plus local orbitals method (FP-APW + lo).

This paper is organized as follows. In Section 2, we briefly described the computational parameters used in the calculations. In Section 3, we discussed the calculated structural properties. Results of elastic constants are presented in Section 4. Electronic band structures are discussed in Section 5. Finally, we give a brief conclusion.

\section{Calculation method}

The first-principle calculations are performed by employing a full-potential (linear) augmented plane wave plus local orbital (FP-(L)APW + lo) [18-20] method, based on density functional theory $[21,22]$ and implemented in the most recent version of the WIEN2K package [23]. The exchange-correlation potential for structural properties was calculated by the local-density approximation (LDA) [24] with and without generalized gradient approximation (GGA) based on Perdew et al. [25], while for electronic properties in addition to that, the Engel-Vosko (GGAEV) [26] scheme was applied. In the FP-(L)APW + lo method, the unit cell is divided into non-overlapping spheres centered at atomic sites (muffin-tin spheres) of radius $R_{\mathrm{MT}}$ and an interstitial region. In the muffin-tin spheres, the Kohn-Sham wave functions are expanded in a linear combination of radial functions time spherical harmonics, and in the remaining space of the unit cell a plane wave basis set is chosen. The basis set inside each muffin-tin sphere is split into core and valence subsets. The core states are treated within the spherical part of the potential only and are assumed to have a spherically symmetric charge density totally confined inside the muffin-tin spheres. The valence part is treated within a potential expanded into spherical harmonics. The valence wave functions inside the spheres are expanded up to $l_{\max }=12$. The $\mathrm{Ba}\left(5 \mathrm{~s}^{2} 5 \mathrm{p}^{6} 6 \mathrm{~s} 2\right), \quad \mathrm{S}\left(3 \mathrm{~s}^{2} 3 \mathrm{p}^{4}\right), \quad \mathrm{Se}\left(3 \mathrm{~d}^{10} 4 \mathrm{~s}^{2} 4 \mathrm{p}^{4}\right), \quad \mathrm{Te}\left(4 \mathrm{~d}^{10} 5 \mathrm{~s}^{2} 5 \mathrm{p}^{4}\right)$ and $\operatorname{Po}\left(5 \mathrm{~d}^{10} 6 \mathrm{~s}^{2} 6 \mathrm{p}^{4}\right)$ states are treated as valence electrons. The $R_{\mathrm{MT}}$ are taken to be 3.0, 2.6, 2.8, 3.0 and 3.0 atomic units (a.u.) for $\mathrm{Ba}, \mathrm{S}, \mathrm{Se}, \mathrm{Te}$ and $\mathrm{Po}$, respectively. A plane wave cut-off $K_{\max }=3.5 \mathrm{a}^{-\mathrm{u}^{-1}}$ is chosen for the expansion of the wave functions in the interstitial region. The $k$ integrations over the Brillouin zone is performed up to $10 \times 10 \times 10$ Monkhorst-Pack [27] mesh for both B1 and B2 phases. The self-consistent calculations are considered to be converged when the total energy of the system is stable within $10^{-5} \mathrm{Ry}$.

\section{Structural properties}

In order to calculate the ground states properties of $\mathrm{BaS}$, $\mathrm{BaSe}, \mathrm{BaTe}$ and $\mathrm{BaPo}$, the total energies are calculated in both phases B1 and B2 for different volumes around the equilibrium cell volume $V_{0}$. The plots of calculated total energies versus reduced volume for these compounds in both phases are given in Fig. 1(a). It is seen from these $E-V$ curves, that the B1 structure is the most stable at room conditions, which is consistent with experimental results and other theoretical works [6-16]. The calculated total energies are fitted to the Murnaghan's equation of state [28] to determine the ground state properties such as the equilibrium lattice constant $a_{0}$, the bulk modulus $B_{0}$ and its pressure derivative $B^{\prime}$. The calculated equilibrium parameters $\left(a_{0}, B_{0}\right.$ and $\left.B^{\prime}\right)$ in both structures are given in Table 1 which also contains results of previous calculations as well as the experimental data. It is clearly seen that for 

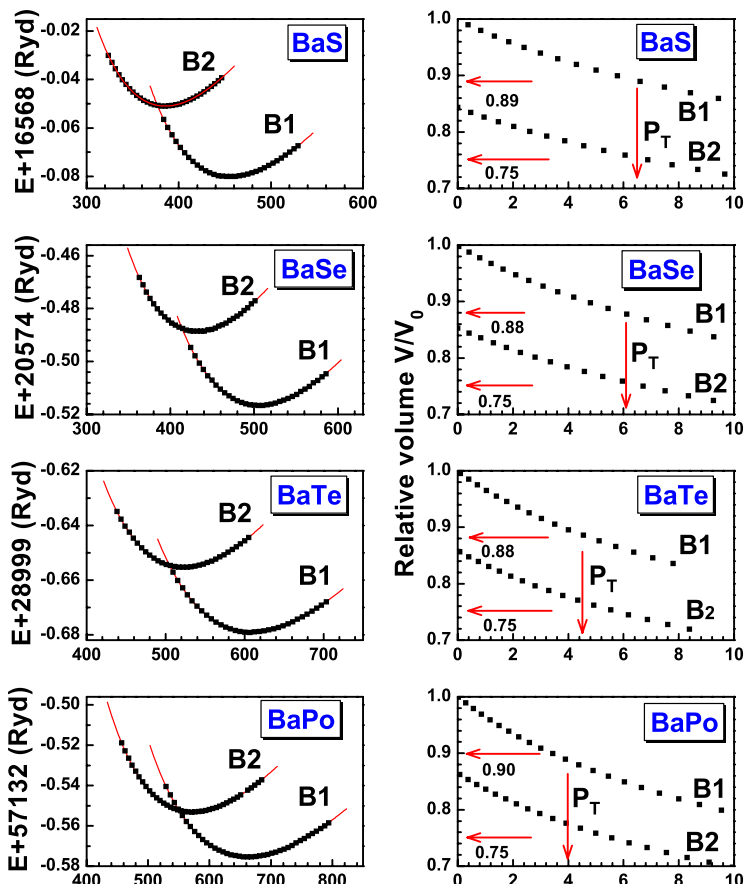

(a) Volume (a.u.)

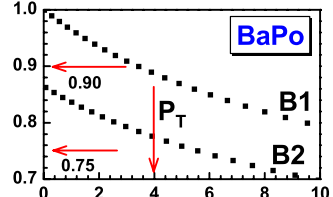

(b) Pressure (GPa)

Fig. 1. (a) Variation of total energies as a function of volume of unit cell for $\mathrm{BaS}, \mathrm{BaSe}, \mathrm{BaTe}$ and BaPo compounds in both structures $\mathrm{B} 1$ and $\mathrm{B} 2$. The solid curve is a least-square fit to Murnaghan's equation of state. (b) The variation of relative volume $V / V_{0}$ of $\mathrm{BaS}, \mathrm{BaSe}, \mathrm{BaTe}$ and $\mathrm{BaPo}$ with pressure in $\mathrm{B} 1$ and $\mathrm{B} 2$ phases. $V_{0}$ is the zero pressure equilibrium volume of the $\mathrm{B} 1$ phase. The arrows mark the calculated transition pressure $P_{\mathrm{T}}$ and the relative volumes at the transition pressure in $\mathrm{B} 1$ and $\mathrm{B} 2$ phases.

all compounds the GGA overestimates the lattice parameter while the LDA underestimates it; these findings are consistent with the general trends of these approximations. Furthermore, the calculated bulk modulus using the LDA approximation are slightly higher than the experimental ones and their values decrease from $\mathrm{BaS}$ to $\mathrm{BaPo}$ for both phases, i.e. from the lower to the higher atomic number. This suggests that $\mathrm{BaPo}$ is more compressible than the other three compounds.

Under compression, the calculation shows that BaS, $\mathrm{BaSe}, \mathrm{BaTe}$ and $\mathrm{BaPo}$ will undergo a structural phase transition from $\mathrm{B} 1$ to $\mathrm{B} 2$ structure. In order to determine the transition pressure at $T=0 \mathrm{~K}$, the enthalpy, $H=E_{0}+$ $P V$, should be calculated [29]. The stable structure at a given pressure is the state for which the enthalpy has its lowest value and the transition pressure is calculated at which the enthalpies for the two phases are equal. Hence the pressure $\left(P_{\mathrm{T}}\right)$ for the structural phase transition from $\mathrm{B} 1$ to $\mathrm{B} 2$ phase is determined by the common tangent of the two $E-V$ curves, B1 and B2 structures. The results of the calculated phase transition parameters are listed in Table 2, together with theoretical and available experimental ones. In BaS, a crystallographic transition from B1 to $\mathrm{B} 2$ is found to be around $6.51 \mathrm{GPa}$ with a volume collapse $\Delta V / V_{0}$ of about $11 \%$ ( $\Delta V$ is the change in volume at transition pressure and $V_{0}$ is the zero pressure equilibrium volume). Experimentally, $\mathrm{BaS}$ has been found to undergo

Table 1

Calculated lattice constant $\left(a_{0}\right)$, bulk modulus $\left(B_{0}\right)$ and its pressure derivative $\left(B^{\prime}\right)$ compared to experiment and other theoretical works for $\mathrm{BaS}, \mathrm{BaSe}, \mathrm{BaTe}$ and $\mathrm{BaPo}$ in $\mathrm{B} 1$ and $\mathrm{B} 2$ structures

\begin{tabular}{|c|c|c|c|c|}
\hline \multirow[t]{2}{*}{ Compounds } & \multicolumn{2}{|c|}{ Present work } & \multirow[t]{2}{*}{ Experiments } & \multirow[t]{2}{*}{ Earlier works } \\
\hline & LDA & GGA & & \\
\hline $\begin{array}{c}B a S(B 1) \\
a_{0}(\AA)\end{array}$ & 6.316 & 6.469 & $6.389^{\mathrm{a}}$ & $\begin{array}{l}6.196^{\mathrm{b}}, \\
6.407^{\mathrm{c}}, 6.276^{\mathrm{c}}\end{array}$ \\
\hline $\begin{array}{l}B_{0}(\mathrm{GPa}) \\
B^{\prime}\end{array}$ & $\begin{array}{l}53.32 \\
4.90\end{array}$ & $\begin{array}{l}42.36 \\
5.81\end{array}$ & $39.42^{\mathrm{a}}$ & $52.46^{\mathrm{b}}$ \\
\hline $\begin{array}{c}B a S(B 2) \\
a_{0}(\AA) \\
B_{0}(\mathrm{GPa}) \\
B^{\prime}\end{array}$ & $\begin{array}{l}3.874 \\
49.50 \\
4.48\end{array}$ & $\begin{array}{l}3.850 \\
45.25 \\
4.38\end{array}$ & $34.02^{\mathrm{a}}$ & $\begin{array}{l}3.706^{\mathrm{b}} \\
60.84^{\mathrm{b}}\end{array}$ \\
\hline $\begin{array}{c}\mathrm{BaSe}(\mathrm{Bl}) \\
\quad a_{0}(\AA)\end{array}$ & 6.511 & 6.696 & $6.595^{\mathrm{d}}$ & $\begin{array}{l}6.42^{\mathrm{b}}, 6.640^{\mathrm{c}} \\
6.477^{\mathrm{c}}\end{array}$ \\
\hline $\begin{array}{l}B_{0}(\mathrm{GPa}) \\
B^{\prime}\end{array}$ & $\begin{array}{l}45.95 \\
4.42\end{array}$ & $\begin{array}{l}36.36 \\
4.72\end{array}$ & $43.4 \pm 2.6^{\mathrm{d}}$ & $45.41^{\mathrm{b}}, 46.8^{\mathrm{d}}$ \\
\hline $\begin{array}{c}B a S e(B 2) \\
a_{0}(\AA) \\
B_{0}(\mathrm{GPa}) \\
B^{\prime}\end{array}$ & $\begin{array}{l}3.874 \\
49.50 \\
4.48\end{array}$ & $\begin{array}{l}4.000 \\
39.42 \\
4.26\end{array}$ & $41.9 \pm 1.4^{\mathrm{d}}$ & $\begin{array}{l}3.471^{\mathrm{a}} ; 3.84^{\mathrm{b}} \\
52.9^{\mathrm{b}}, 48.6^{\mathrm{e}}\end{array}$ \\
\hline $\begin{array}{c}B a T e(B 1) \\
\quad a_{0}(\AA)\end{array}$ & 6.920 & 7.121 & $7.007^{\mathrm{f}}, 6.9989^{\mathrm{d}}$ & $\begin{array}{l}6.95^{\mathrm{b}}, 7.06^{\mathrm{g}}, 6.63^{\mathrm{g}} \\
6.989^{\mathrm{c}}, 6.869^{\mathrm{c}}\end{array}$ \\
\hline $\begin{array}{l}B_{0}(\mathrm{GPa}) \\
B^{\prime}\end{array}$ & $\begin{array}{l}35.68 \\
4.51\end{array}$ & $\begin{array}{l}28.70 \\
5.42\end{array}$ & $\begin{array}{l}29.4^{\mathrm{c}} \\
7.4^{\mathrm{c}}\end{array}$ & $\begin{array}{l}33.75^{\mathrm{b}}, 35.40^{\mathrm{e}} \\
4.64^{\mathrm{e}}, 4.27^{\mathrm{g}}\end{array}$ \\
\hline $\begin{array}{c}B a T e(B 2) \\
a_{0}(\AA) \\
B_{0}(\mathrm{GPa}) \\
B^{\prime}\end{array}$ & $\begin{array}{l}4.122 \\
40.04 \\
4.11\end{array}$ & $\begin{array}{l}4.263 \\
31.81 \\
4.26\end{array}$ & $\begin{array}{l}27.5^{\mathrm{c}} \\
4.60^{\mathrm{f}}\end{array}$ & $\begin{array}{l}3.621^{\mathrm{a}}, 4.19^{\mathrm{b}} \\
39.41^{\mathrm{b}}, 150.43^{\mathrm{a}} \\
6.0^{\mathrm{g}}, 4.65^{\mathrm{e}}\end{array}$ \\
\hline $\begin{array}{c}\mathrm{BaPo}(\mathrm{BI}) \\
a_{0}(\AA) \\
B_{0}(\mathrm{GPa}) \\
B^{\prime}\end{array}$ & $\begin{array}{l}7.117 \\
32.19 \\
4.52\end{array}$ & $\begin{array}{l}7.334 \\
25.70 \\
4.18\end{array}$ & $7.119^{c}$ & $7.046^{\mathrm{c}}, 6.95^{\mathrm{c}}$ \\
\hline $\begin{array}{c}B a P o(B 2) \\
a_{0}(\AA) \\
B_{0}(\mathrm{GPa}) \\
B^{\prime}\end{array}$ & $\begin{array}{l}4.22 \\
36.15 \\
4.46\end{array}$ & $\begin{array}{l}4.394 \\
28.70 \\
4.23\end{array}$ & & \\
\hline
\end{tabular}

${ }^{a}$ Ref. [9].

${ }^{b}$ Ref. [12].

${ }^{c}$ Ref. [5].

${ }^{\mathrm{d}}$ Ref. [10].

e Ref. [14].

${ }^{f}$ Ref. [8].

${ }^{\mathrm{g}}$ Ref. [15].

first-order phase transition from $\mathrm{B} 1$ structure to the $\mathrm{B} 2$ structure at pressure equal to $6.50 \mathrm{GPa}[6]$. The calculated pressure transition from $\mathrm{B} 1$ phase to $\mathrm{B} 2$ phase in $\mathrm{BaSe}$ is found to be equal to $6.02 \mathrm{GPa}$. This phase transition is accompanied by a volume collapse equal to $12 \%$ for BaSe. The measured $P_{\mathrm{T}}$ for BaSe is found to be around 6.0 GPa [7]. Our calculations show that the phase transition pressure for BaTe is $4.52 \mathrm{GPa}$ with a volume collapse equal to $12 \%$. The experimental study shows that the phase transition pressure is $4.8 \mathrm{GPa}[8]$. For $\mathrm{BaPo}$ we have found that $P_{\mathrm{T}}$ is around $4.0 \mathrm{GPa}$ with a volume collapse equal to $12 \%$. 
Table 2

Calculated values of the transition pressure $P_{\mathrm{T}}$, the transition volumes $V_{\mathrm{T}}$, the volume change at the transition pressure $\Delta V$ (in \%) for $\mathrm{BaS}$, BaSe, $\mathrm{BaTe}$ and $\mathrm{BaPo}$

\begin{tabular}{|c|c|c|c|c|c|}
\hline & $\begin{array}{l}P_{\mathrm{T}} \\
(\mathrm{GPa})\end{array}$ & $\begin{array}{l}V_{\mathrm{T}}(\mathrm{B} 1) / \\
V_{0}(\mathrm{~B} 1)\end{array}$ & $\begin{array}{l}V_{\mathrm{T}}(\mathrm{B} 2) / \\
V_{0}(\mathrm{~B} 1)\end{array}$ & $\begin{array}{l}V_{0}(\mathrm{~B} 2) / \\
V_{0}(\mathrm{~B} 1)\end{array}$ & $\Delta V / V_{0}$ \\
\hline \multicolumn{6}{|l|}{$B a S$} \\
\hline Present & 6.51 & 0.890 & 0.753 & 0.845 & 11.0 \\
\hline Expt. & $6.5^{\mathrm{a}}$ & 0.896 & 0.773 & & 13.7 \\
\hline \multirow[t]{2}{*}{ Others } & $6.025^{\mathrm{b}}$ & $0.873^{\mathrm{b}}$ & $0.773^{\mathrm{b}}$ & & $13.8^{\mathrm{b}}$ \\
\hline & $7.3^{\mathrm{c}}$ & & & & $14.29^{\mathrm{c}}$ \\
\hline \multicolumn{6}{|l|}{ BaSe } \\
\hline Present & 6.02 & 0.88 & 0.759 & 0.854 & 12.0 \\
\hline Expt. & $6.0^{\mathrm{d}}$ & & & & 13.9 \\
\hline \multirow[t]{2}{*}{ Others } & $5.2^{\mathrm{b}}$ & $0.88^{\mathrm{b}}$ & $0.762^{\mathrm{b}}$ & & $13.4^{\mathrm{b}}$ \\
\hline & $5.6^{\mathrm{e}}$ & $0.89^{\mathrm{e}}$ & $0.76^{\mathrm{e}}$ & & $14.6^{\mathrm{e}}$ \\
\hline \multicolumn{6}{|l|}{ BaTe } \\
\hline Present & 4.52 & 0.886 & 0.753 & 0.859 & 12.0 \\
\hline Expt. & $4.8^{\mathrm{f}}$ & & & & 13.2 \\
\hline \multirow[t]{4}{*}{ Others } & $3.95^{\mathrm{b}}$ & $0.894^{\mathrm{b}}$ & $0.798^{\mathrm{b}}$ & & $10.7^{\mathrm{b}}$ \\
\hline & $3.2^{\mathrm{e}}$ & $0.925^{\mathrm{e}}$ & $0.8^{\mathrm{e}}$ & & $13.5^{\mathrm{e}}$ \\
\hline & $3.8^{\mathrm{f}}$ & $0.85^{\mathrm{g}}$ & $0.78^{\mathrm{g}}$ & & \\
\hline & $5.942^{\mathrm{g}}$ & $0.77^{\mathrm{g}}$ & $0.66^{\mathrm{g}}$ & & \\
\hline \multicolumn{6}{|l|}{$\mathrm{BaPo}$} \\
\hline Present & 4.00 & 0.901 & 0.772 & 0.864 & 10.0 \\
\hline \multicolumn{6}{|c|}{$\begin{array}{l}\text { Comparison is made with the available theoretical and experimental da } \\
\text { a Ref. [9]. }\end{array}$} \\
\hline \multicolumn{6}{|c|}{${ }^{\mathrm{b}}$ Ref. [12]. } \\
\hline \multicolumn{6}{|c|}{${ }^{\mathrm{c}}$ Ref. [16]. } \\
\hline \multicolumn{6}{|c|}{${ }^{\mathrm{d}}$ Ref. [7]. } \\
\hline \multicolumn{6}{|c|}{${ }^{\mathrm{e}}$ Ref. [10]. } \\
\hline \multicolumn{6}{|c|}{${ }^{\mathrm{f}}$ Ref. [14]. } \\
\hline \multicolumn{6}{|c|}{${ }^{\mathrm{g}}$ Ref. [15] } \\
\hline \multicolumn{6}{|c|}{$\begin{array}{l}\text { For } \mathrm{BaPo} \text {, there is no experimental data available to us to } \\
\text { check our results. It is interesting to note a linear decrease } \\
\text { of the transition pressure with the increase in the size of the } \\
\text { chalcogen atoms. }\end{array}$} \\
\hline
\end{tabular}

\section{Elastic constants}

The elastic properties define the properties of material that undergoes stress, deforms and then recovers and returns to its original shape after stress ceases. These properties play an important part in providing valuable information about the binding characteristic between adjacent atomic planes, anisotropic character of binding and structural stability. Hence, to study the stability of these compounds in $\mathrm{NaCl}-\mathrm{B} 1$ and $\mathrm{CsCl}-\mathrm{B} 2$ structures, we have calculated the elastic constants at normal and under hydrostatic pressure by using the method developed recently by Thomas Charpin and integrated it in the WIEN2K package [23]. The elastic moduli require knowledge of the derivative of the energy as a function of lattice strain. In the case of cubic system, there are only three independent elastic constants, namely, $C_{11}, C_{12}$ and $C_{44}$. So a set of three equations is needed to determine all the constants, which means that three types of strain must be applied to the starting crystals. The first equation involves calculating the bulk modulus $B_{0}$, which is related to the elastic constants by the following formula:

$B_{0}=\left(C_{11}+2 C_{12}\right) / 3$

The second one involves performing volume-conservative tetragonal strain tensor:

$$
\left[\begin{array}{ccc}
\delta & 0 & 0 \\
0 & \delta & 0 \\
0 & 0 & \frac{1}{(1+\delta)^{2}}-1
\end{array}\right]
$$

Application of this strain has an effect on the total energy from its unstrained value as follows:

$E(\delta)=E(0)+3\left(C_{11}-C_{12}\right) V_{0} \delta_{+}^{2} O\left(\delta^{3}\right)$,

where $V_{0}$ is the volume of the unit cell. Finally, for the last type of deformation, we use the volume-conserving rhombohedral strain tensor given by

$\frac{\delta}{3}\left[\begin{array}{lll}1 & 1 & 1 \\ 1 & 1 & 1 \\ 1 & 1 & 1\end{array}\right]$

This changes the total energy to

$E(\delta)=E(0)+\frac{1}{6}\left(C_{11}+2 C_{12}+4 C_{44}\right) V_{0} \delta^{2}+O\left(\delta^{3}\right)$

In our calculations, we consider only small lattice distortions in order to remain within the elastic domain of the crystal. In the present study, five sets of $E(\delta)$ for the four compounds ( $\mathrm{BaS}, \mathrm{BaSe}, \mathrm{BaTe}$ and $\mathrm{BaPo}$ ) in both $\mathrm{B} 1$ and B2 phases are calculated.

To study the stability of these compounds in the B1 and B2 structures, we have calculated the elastic constants at the equilibrium lattice and compared our results to the stability criteria $[30,31]$ using the following relations:

$C_{11}-C_{12}>0, \quad C_{44}>0, \quad B_{0}>0$

We have found that in both structures, these criteria are satisfied, indicating that both phases are elastically stable. To predict the metastability of the $\mathrm{CsCl}-\mathrm{B} 2$ structure we need to perform phonon frequency calculations. The calculated values of elastic constants in both $\mathrm{B} 1$ and $\mathrm{B} 2$ phases are listed in Table 3. These compounds, involving barium as common cation, have comparatively higher lattice parameters and smaller bulk moduli than other calcium chalcogenides [32]. This implies that the elastic constants are relatively small. Moreover, we expect that these elastic constants computed using our bulk modulus (Eq. (1)) overestimate the corresponding experiment values because the experimental bulk modulus is less than ours. Since the true (experimental) values of the elastic constants are not available, the magnitude of this overestimation is difficult to estimate. It may be assumed to be small, since the calculation is performed with a large number of $k$-points and a large number of plane waves. We consider the present elastic constants as a prediction study for these compounds, hoping that our present work will stimulate some other works 
Table 3

The LDA calculated elastic constants $\left(C_{11}, C_{12}\right.$ and $\left.C_{44}\right)$ for barium chalcogenides $\mathrm{BaS}, \mathrm{BaSe}, \mathrm{BaTe}$ and $\mathrm{BaPo}$ in $\mathrm{B} 1$ and $\mathrm{B} 2$ structures

\begin{tabular}{|c|c|c|c|}
\hline Compounds & $C_{11}(\mathrm{GPa})$ & $C_{12}(\mathrm{GPa})$ & $C_{44}(\mathrm{GPa})$ \\
\hline \multicolumn{4}{|l|}{$\begin{array}{l}\mathrm{NaCl}(\mathrm{Bl}) \\
\mathrm{BaS}\end{array}$} \\
\hline Present & 115 & 17 & 18 \\
\hline \multicolumn{4}{|l|}{ BaSe } \\
\hline Present & 104 & 14 & 15 \\
\hline \multicolumn{4}{|l|}{ BaTe } \\
\hline Present & 87 & 09 & 12 \\
\hline \multicolumn{4}{|l|}{$\mathrm{BaPo}$} \\
\hline Present & 81 & 07 & 09 \\
\hline \multicolumn{4}{|l|}{$\mathrm{CsCl}(\mathrm{B} 2)$} \\
\hline \multicolumn{4}{|l|}{$B a S$} \\
\hline Present & 133 & 21 & 22 \\
\hline \multicolumn{4}{|l|}{$B a S e$} \\
\hline Present & 116 & 16 & 31 \\
\hline \multicolumn{4}{|l|}{ BaTe } \\
\hline Present & 90 & 15 & 23 \\
\hline \multicolumn{4}{|l|}{$\mathrm{BaPo}$} \\
\hline Present & 82 & 13 & 12 \\
\hline
\end{tabular}

on these compounds. The elastic constants increase in magnitude as a function of the anion chemical identity as one moves upwards within period VI, i.e. from Po to S. For barium chalcogenides, with the increase of atomic number, the ionic characteristic decreases and the metallic one increases from a $\mathrm{Ba}-\mathrm{S}$ bond to $\mathrm{Ba}-\mathrm{Po}$ bond, accompanied by a decrease of the magnitude of elastic constants. We note a linear increase of the elastic constants with the decrease of the nearest-neighbor distance from BaPo to BaS.

Now we are interested to study the pressure dependence of the elastic properties. In Fig. 2, we present the variation of the elastic constants $\left(C_{11}, C_{12}, C_{44}\right)$ and the bulk modulus $B_{0}$ of $\mathrm{BaS}, \mathrm{BaSe}, \mathrm{BaTe}$ and $\mathrm{BaPo}$ with respect to the variation of pressure. We clearly observe a linear dependence in all curves of these compounds in the considered range of pressure, confirming the idea of Polian et al. [33], Harrera-Cabrira et al. [34] and Kanoun et al. [35] of the no responsibility of the soft acoustic mode on the phase transition. In Table 4, we listed our results for the pressure derivatives $\partial C_{11} / \partial P, \partial C_{12} / \partial P, \partial C_{44} / \partial P$ and, $\partial B_{0} / \partial P$, for $\mathrm{BaS}, \mathrm{BaSe}, \mathrm{BaTe}$ and BaPo. It is easy to observe that the elastic constants $C_{11}, C_{12}$ and bulk modulus $B_{0}$ increase when the pressure is enhanced. Moreover the shown shear mode modulus $C_{44}$ decreases linearly with the increasing of pressure. To our knowledge no experimental or theoretical data for the pressure derivative of elastic constants of these compounds are given in the literature. Then, our results can serve as a prediction for future investigations.

\section{Electronic structure}

Fig. 3, shows the calculated band structures at equilibrium volume for $\mathrm{BaTe}$ in $\mathrm{NaCl}$ phase within GGA-EV as a prototype since the band profiles are quite similar for all four compounds, with a small difference. The overall band profiles are in fairly good agreement with previous theoretical results $[5,12,14,16,17]$. In all cases, the valence band maximum (VBM) and conduction band minimum
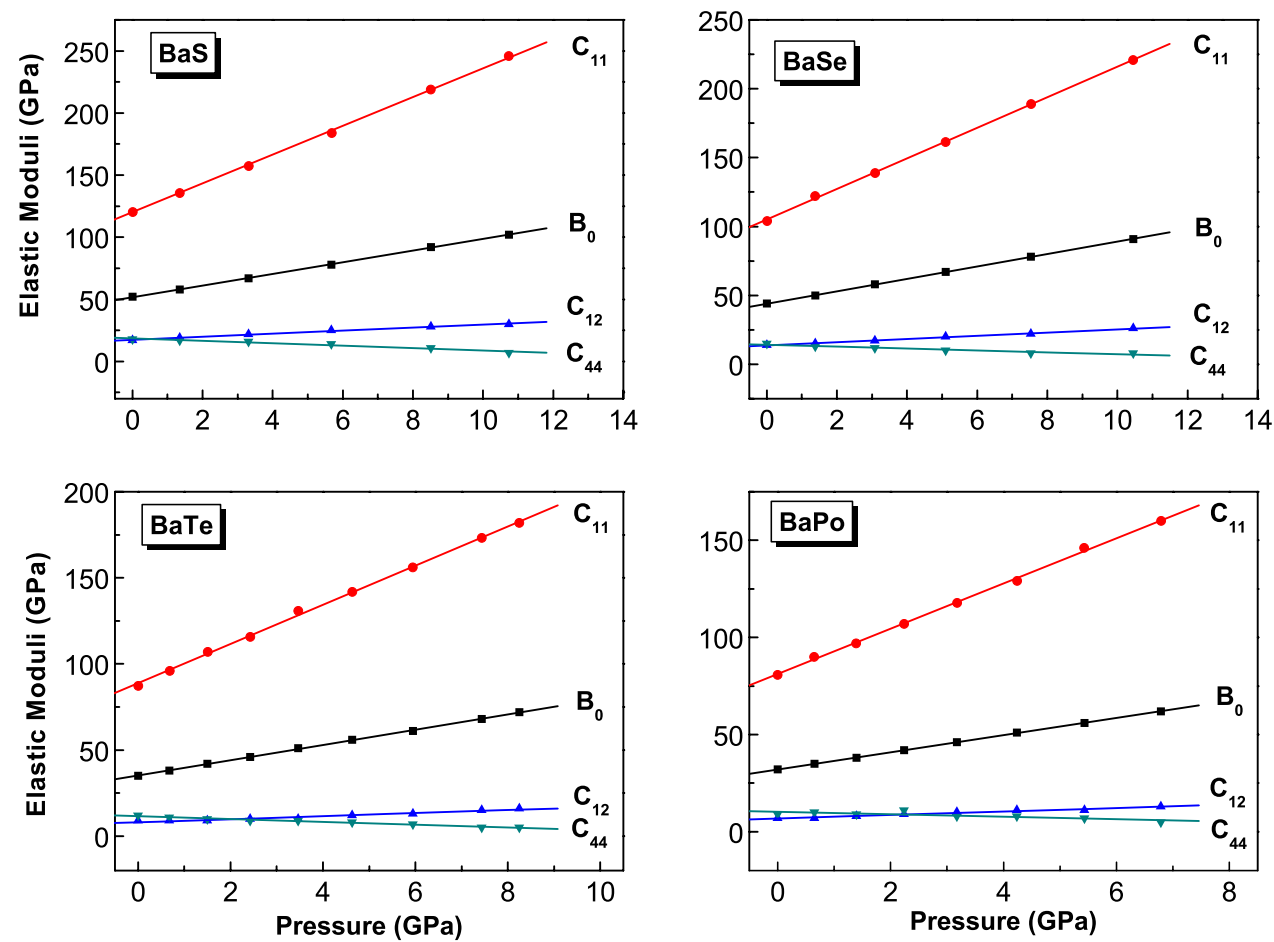

Fig. 2. Calculated pressure dependence of elastic constants $\mathrm{C}_{i j}$ and bulk modulus $B_{0}$ for $\mathrm{BaS}$, BaSe, BaTe and BaPo in $\mathrm{B} 1$ phase. 
Table 4

Pressure dependence of the bulk modulus and the elastic constants $\left(C_{11}\right.$, $C_{12}$ and $C_{44}$ ) of $\mathrm{BaS}, \mathrm{BaSe}, \mathrm{BaTe}$ and BaPo in $\mathrm{B} 1$ phase

\begin{tabular}{lcccc}
\hline Compounds & $\frac{\partial B_{0}}{\partial P}$ & $\frac{\partial C_{11}}{\partial P}$ & $\frac{\partial C_{12}}{\partial P}$ & $\frac{\partial C_{44}}{\partial P}$ \\
\hline $\begin{array}{l}\text { BaS } \\
\begin{array}{l}\text { Present } \\
\text { BaSe }\end{array}\end{array}$ & 4.91 & 11.60 & 1.21 & -0.98 \\
$\begin{array}{l}\text { Present } \\
\text { BaTe }\end{array}$ & 4.42 & 11.11 & 1.16 & -0.69 \\
$\begin{array}{l}\text { Present } \\
\text { BaPo }\end{array}$ & 4.51 & 11.38 & 0.89 & -0.82 \\
\begin{tabular}{l} 
Present \\
\hline
\end{tabular} & 4.52 & 11.64 & 0.88 & -0.64 \\
\hline
\end{tabular}

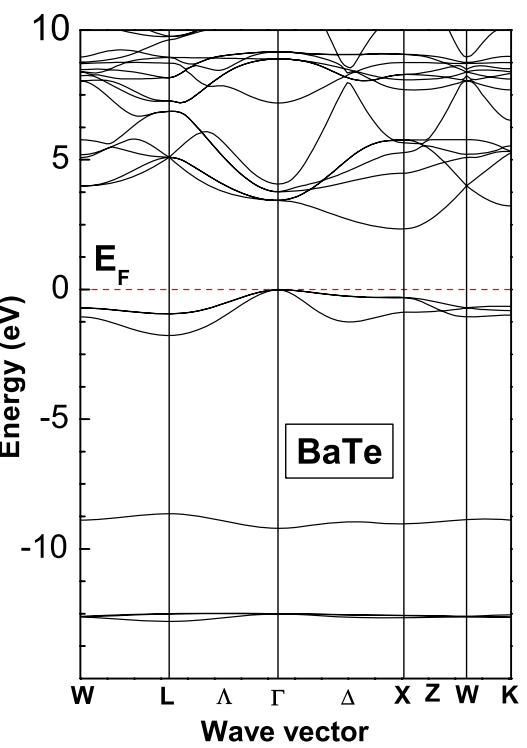

Fig. 3. Band structure of BaTe calculated along high symmetry directions within GGA-EV scheme in B1 phase at equilibrium volume.

(CBM) occurs at $\Gamma$ and $X$ points, respectively. Thus the energy gap is indirect between the top of the (anion p) valence band and the bottom of the (cation d) conduction. Note that the anion $p$ bands shift up in energy going from the sulphide to the Po. This is the normal behaviour related to the increase of the lattice parameters, which was also found for II-VI compounds [36-38]. The calculated values of band gaps and valence band widths for the four compounds studied in present work are given in Table 5 along with the experimental values and the other theoretical works available in literature. The main features to note from this table are as follows:

1. Our calculated values using LDA and GGA are in agreement with similar computational works $[5,12,15]$.

2. The values of calculated band gaps with GGA-EV are in better agreement with experiment [2,3], compared to other theoretical works which are based on LDA and GGA.

It is well known that the LDA and the GGA usually underestimate the energy gap $[39,40]$. This is mainly due to
Table 5

Results of band gap and upper-valence bandwidth of $\mathrm{BaS}, \mathrm{BaSe}, \mathrm{BaTe}$ and BaPo in B1 phase

\begin{tabular}{|c|c|c|c|c|}
\hline Compounds & Method & $\begin{array}{l}\text { Exchange } \\
\text { correlation } \\
\mathrm{XC}\end{array}$ & $\begin{array}{l}\text { Energy } \\
\text { gap }(\Gamma-X) \\
(\mathrm{eV})\end{array}$ & Bandwidth $(\mathrm{eV})$ \\
\hline \multicolumn{5}{|l|}{$B a S$} \\
\hline \multirow[t]{3}{*}{ Present work } & FP-LAPW & LDA & 1.94 & 2.018 \\
\hline & & GGA & 2.26 & 1.789 \\
\hline & & GGA-EV & 3.07 & 1.451 \\
\hline \multirow[t]{2}{*}{ Cal. (DFT) } & PP-PW & LDA & $1.83[5]$ & \\
\hline & & GGA & $2.17[5]$ & \\
\hline Cal. (DFT) & TB-LMTO & GGA & $2.30[12]$ & \\
\hline Expt. & & & $3.88[2]$ & \\
\hline Expt. & & & $3.90[3]$ & \\
\hline \multicolumn{5}{|l|}{ BaSe } \\
\hline \multirow[t]{3}{*}{ Present work } & FP-LAPW & LDA & 1.74 & 2.176 \\
\hline & & GGA & 2.04 & 1.921 \\
\hline & & GGA-EV & 2.77 & 1.601 \\
\hline \multirow[t]{2}{*}{ Cal. (DFT) } & PP-PW & LDA & $1.63[5]$ & \\
\hline & & GGA & $2.02[5]$ & \\
\hline Cal. (DFT) & TB-LMTO & GGA & $2.01[12]$ & \\
\hline Expt. & & & $3.58[2]$ & \\
\hline Expt. & & & $3.60[3]$ & \\
\hline \multicolumn{5}{|l|}{ BaTe } \\
\hline \multirow[t]{3}{*}{ Present work } & FP-LAPW & LDA & 1.28 & 2.356 \\
\hline & & GGA & 1.68 & 2.071 \\
\hline & & GGA-EV & 2.36 & 1.75 \\
\hline \multirow[t]{2}{*}{ Cal. (DFT) } & PP-PW & LDA & $1.28[5]$ & \\
\hline & & GGA & $1.65[5]$ & \\
\hline Cal. (DFT) & TB-LMTO & GGA & $1.58[12]$ & \\
\hline Cal. (DFT) & FP-LAPW & GGA & $1.70[15]$ & \\
\hline Expt. & & & $3.08[2]$ & \\
\hline Expt. & & & $3.10[3]$ & \\
\hline \multicolumn{5}{|l|}{$\mathrm{BaPo}$} \\
\hline \multirow[t]{3}{*}{ Present work } & FP-LAPW & LDA & 1.22 & 02.478 \\
\hline & & GGA & 1.49 & 2.18 \\
\hline & & GGA-EV & 2.13 & 1.86 \\
\hline \multirow[t]{2}{*}{ Cal. (DFT) } & PP-PW & LDA & $1.01[5]$ & \\
\hline & & GGA & $1.45[5]$ & \\
\hline
\end{tabular}

the fact that they have simple forms that are not sufficiently flexible for accurately reproducing both exchange-correlation energy and its charge derivative. Engel and Vosko, by considering this shortcoming constructed a new functional form of the GGA which has been designed to give better exchange-correlation potential at the expense of less agreement as regards exchange energy. This approach, which is called the GGA-EV, yields a better band splitting and some other properties which mainly depend on the accuracy of the exchange-correlation potential. On the other hand, in this method, the quantities which depend on an accurate description of the exchange energy $E_{\mathrm{x}}$ such as equilibrium volumes and the bulk modulus, are in poor agreement with experiment. The values of calculated band gaps with GGA-EV are a significant improvement over the earlier results based on LDA and GGA and closer to the experimental values.

In view of Table 5 , it is clear that the width of the upper valence band increases as we increase the nuclear charge of the anion for different approximations, indicating that the wave function is more localized in going from $\mathrm{BaPo}$ to $\mathrm{BaS}$. 

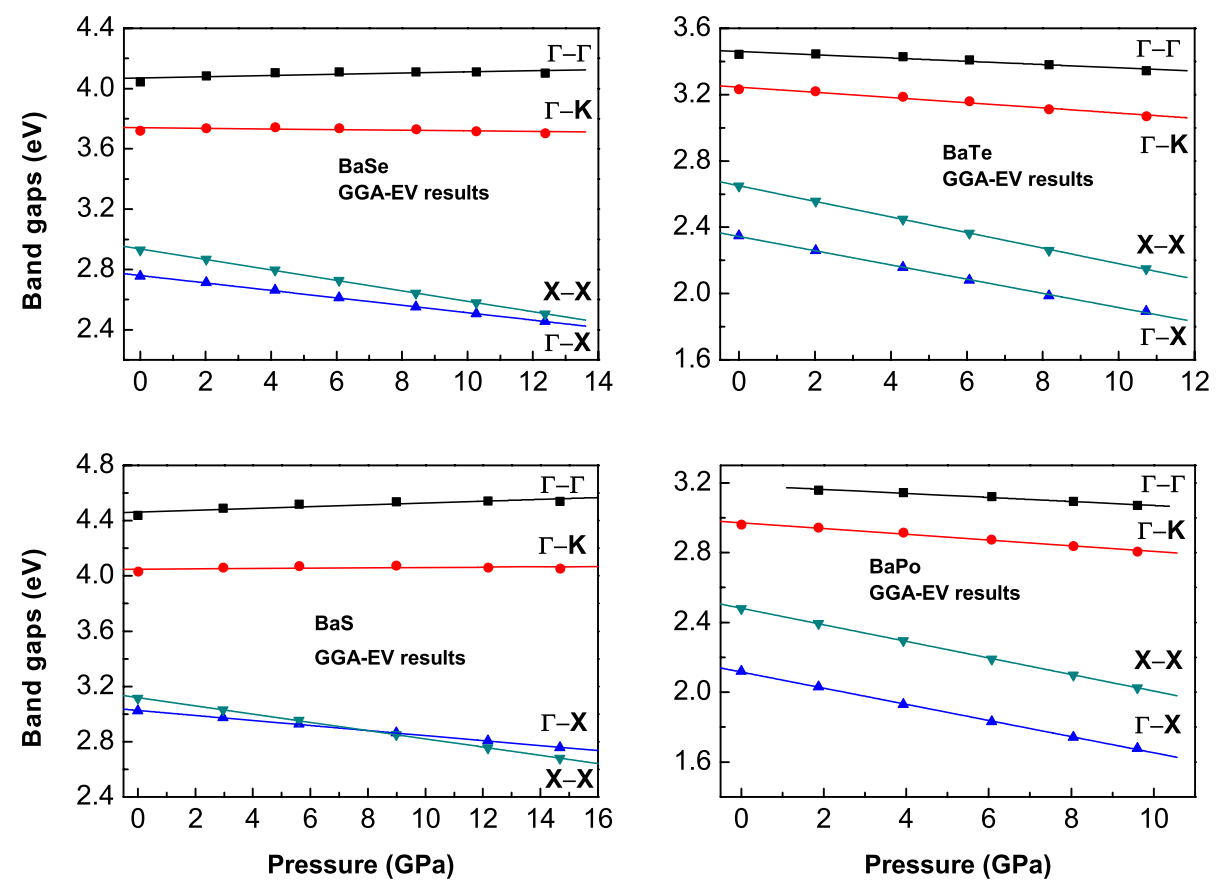

Fig. 4. Plots of the calculated band gaps of $\mathrm{BaS}, \mathrm{BaSe}, \mathrm{BaTe}$ and BaPo versus pressure in $\mathrm{B} 1$ phase.

This is in line with the usual trend in which the valence band states become more localized as a material becomes less covalent and more ionic. Another obvious different character is that the indirect band gap increases as we decrease the nuclear charge of the anion. It also implies that the interaction between anion and cation atoms is weekly covalent in $\mathrm{BaS}$ than BaPo. This assumption along with the recent study of Lin et al. [5] on the ionic character in chemical bond in these compounds.

The pressure-induced energy shifts for the optical transition related to the direct and indirect band gaps for the $\mathrm{NaCl}-\mathrm{B} 1$ phase for these compounds are shown in Fig. 4, where the solid line are a linear least-square fit to the calculated data. Results of the linear pressure coefficients are listed in Table 6. In view of Fig. 4 and Table 6, it is clear that the direct and indirect band gap $(X-X)$ and $(\Gamma-X)$ decrease with applied pressure in all compounds. Whereas, the direct energy gap $(\Gamma-\Gamma)$ enhanced with pressure in $\mathrm{BaS}$ and BaSe. A crossover between the indirect gap $(\Gamma-X)$ and the direct gap $(X-X)$ curves occurs at about $7.9 \mathrm{GPa}$ for

Table 6

Calculated linear pressure coefficients of important band gap for BaS, $\mathrm{BaSe}, \mathrm{BaTe}$ and $\mathrm{BaPo}$ compounds in NaCl-B1 phase using GGA-EV. $E_{i}(p)=E_{i}(0)+b p, b=\partial E_{i} / \partial p$ in $10^{-2} \mathrm{eV} \mathrm{GPa}^{-1}$

\begin{tabular}{lcccc}
\hline & $\Gamma-\Gamma$ & $X-X$ & $\Gamma-K$ & $\Gamma-X$ \\
\hline BaS & & & & \\
$\begin{array}{l}\text { Present } \\
\text { BaSe }\end{array}$ & 0.66 & -2.98 & 0.12 & -1.82 \\
$\begin{array}{l}\text { Present } \\
\text { BaTe }\end{array}$ & 0.89 & -3.47 & -0.20 & -2.47 \\
$\begin{array}{l}\text { Present } \\
\text { BaPo }\end{array}$ & -0.98 & -4.69 & -1.57 & -4.29 \\
Present & -1.15 & -4.75 & -1.64 & -4.63 \\
\hline
\end{tabular}

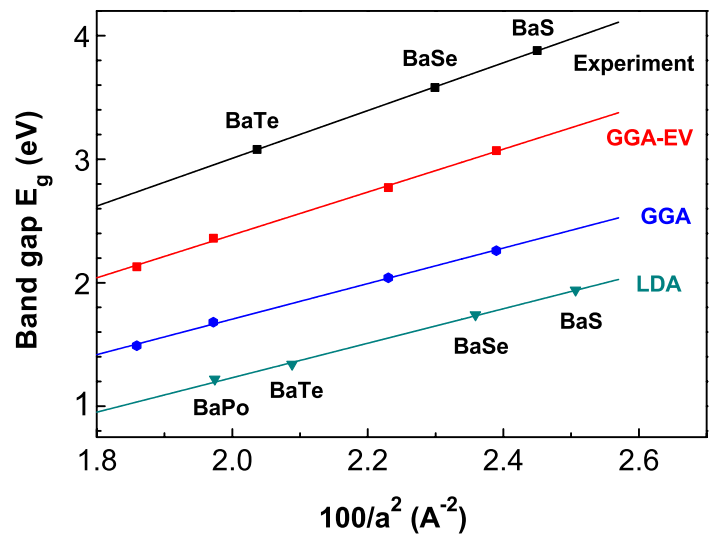

Fig. 5. The measured and calculated band gap (using LDA, GGA and GGA-EV) versus $100 / a^{2}$ (where $a$ is the equilibrium lattice constant) for rock-salt barium chalcogenides $\mathrm{BaS}, \mathrm{BaSe}, \mathrm{BaTe}$ and $\mathrm{BaPo}$.

$\mathrm{BaS}$, resulting in the energy minimum of direct gap for this compound. We have neither theoretical nor experimental values of the pressure coefficients to compare with them; we leave these results as references for future investigation.

Using experimental data of $\mathrm{BaS}, \mathrm{BaSe}$ and BaTe, Dalven [41] summarized an empirical relationship that band gaps of barium chalcogenides were linear with $1 / a^{2}$, where $a$ was the lattice constant of crystal. As it is shown in Fig. 5, our calculated band gaps for $\mathrm{BaS}, \mathrm{BaSe} \mathrm{BaTe}$ and $\mathrm{BaPo}$ using the LDA, the GGA and the GGA-EV methods are linear with $1 / a^{2}$.

\section{Conclusion}

Employing the FP-APW + lo method we have studied the structural, elastic and electronic properties of $\mathrm{BaS}$, 
BaSe, BaTe and BaPo compounds. A summary of our results follows:

1. The calculated structural properties are in a good agreement with the available experimental and theoretical data.

2. The results regarding the high pressure structural phase transition are in a good agreement with the experimental results.

3. We are not aware of any experimental or theoretical data for the elastic properties of these compounds in both B1 and B2 phases and so our calculations can be used to cover this lack of data for these compounds.

4. Our calculated band gaps, using LDA and GGA, are in a good agreement with the other computational works, but lower than the experimental work. The band gaps, calculated with the GGA-EV, are much better than LDA and GGA and close the experimental results.

5. We are not aware of any experimental or theoretical data for the pressure-induced energy shifts for these compounds in B1 structure so our calculations can be used to cover this lack of data.

\section{Acknowledgements}

The first and second authors (A. B.) and (R. K.) wishes to acknowledge the help of Professor F. El Haj Hassen (Elhadath University, Lebanon) and Doctor Rashid Ahmed (Punjab University, Pakistan).

\section{References}

[1] K.L. Heng, S.J. Chua, P. Wu, Chem. Mat. 12 (2000) 1648.

[2] R.J. Zolweg, Phys. Rev. 11 (1958) 113.

[3] G.A. Saum, E.B. Hensley, Phys. Rev. 113 (1959) 1019.

[4] Y. Kaneko, K. Morimoto, T. Koda, J. Phys. Jpn. Soc. 52 (1985) 8385.

[5] G.Q. Lin, H. Gong, P. Wu, Phys. Rev. B 71 (2005) 85203.

[6] S.T. Weir, Y.K. Vohra, A.L. Ruoff, Phys. Rev. B 33 (1986) 4221.

[7] T.A. Grzybowki, A.L. Ruoff, Phys. Rev. B 27 (1983) 6502.

[8] T.A. Grzybowki, A.L. Ruoff, Phys. Rev. Lett. 53 (1984) 489.

[9] S. Yamaoka, O. Shimomuro, H. Nakasawa, O. Fukunaga, Solid State Commun. 33 (1980) 87.

[10] S.H. Wei, H. Krakauer, Phys. Rev. Lett. 55 (1985) 1200.

[11] A.E. Carlsson, J.W. Wilkins, Phys. Rev. B 29 (1984) 5836.
[12] G. Kalpana, B. Palanivel, M. Rajagopalan, Phys. Rev. B 50 (1994) 12318.

[13] P.K. Jha, U.K. Sakalle, S.P. Sanyal, J. Phys. Chem. Solids 59 (1998) 1633.

[14] K. Syassen, N.E. Christensen, H. Winzen, K. Fischer, J. Evers, Phys. Rev. B 35 (1987) 4052.

[15] H. Akbarzadeh, M. Dadsetani, M. Mehrani, Comput. Mater. Sci. 17 (2000) 81.

[16] R. Khenata, M. Sahnoun, H. Baltache, M. Rérat, D. Rached, M. Driz, B. Bouhafs, Physica B 371 (2006) 12.

[17] A. Pouraghazi, M. Dadestani, Physica B 370 (2005) 35.

[18] G.K.H. Madsen, P. Plaha, K. Schwarz, E. Sjöstedt, L. Nordström, Phys. Rev. B 64 (2001) 195134.

[19] E. Sjöstedt, L. Nordström, D.J. Singh, Solid State Commun. 15 (2000) 15.

[20] K. Schwarz, P. Blaha, G.K.H. Madsen, Comput. Phys. Commun. 147 (2002) 71.

[21] P. Hohenberg, W. Kohn, Phys. Rev. B 136 (1964) 64.

[22] W. Kohn, L.J. Sham, Phys. Rev. A 140 (1965) 1133.

[23] P. Blaha, K. Schwarz, G.K.H. Madsen, D. Kvasnicka and J. Luitz, WIEN2k, An Augmented Plane Wave + Local Orbitals Program for Calculating Crystal Properties (Karlheinz Schwarz, Techn. Universität Wien, Austria), 2001, ISBN 3-9501031-1-2.

[24] J.P. Perdew, Y. Wang, Phys. Rev. B 45 (1992) 13244.

[25] J.P. Perdew, S. Burke, M. Ernzerhof, Phys. Rev. Lett. 77 (1996) 3865.

[26] E. Engel, S.H. Vosko, Phys. Rev. B 47 (1993) 13164.

[27] H.J. Monkhorst, L.D. Pack, Phys. Rev. B 13 (1976) 5188.

[28] F.D. Murnaghan, Proc. Natl. Acad. Sci. USA 30 (1944) 244.

[29] W. Wettling, J. Windscheif, Solid State Commun. 50 (1984) 22.

[30] M. Born, K. Huang, Dynamical Theory of Crystal Lattices, Clarendon, Oxford, 1954.

[31] J. Wang, S. Yip, Phys. Rev. Lett. 71 (1993) 4182.

[32] Z. Charifi, H. Baaziz, F. El Hadj Hassen, N. Bouarissa, J. Phys. Condens. Matter 17 (2005) 4083.

[33] A. Polian, M. Grimsditch, Phys. Rev. B 60 (1999) 1468.

[34] M.J. Harrera-Cabrera, P. Rodríguez-Hernández, A. Muñoz, Phys. Status Solidi B 223 (2001) 411.

[35] M.B. Kanoun, A.E. Merad, G. Merad, J. Cibert, H. Aourag, SolidState Electron. 48 (2004) 1601;

M.B. Kanoun, S. Goumri-Said, A.E. Merad, G. Merad, J. Cibert, H. Aourag, Semicond. Sci. Technol. 19 (2004) 1220.

[36] S.-H. Wei, A. Zunger, Phys. Rev. B 53 (1996) R10457.

[37] D. Rached, M. Rabah, N. Benkhettou, R. Khenata, B. Soudini,Y. AlDouri, H. Baltache, Comput. Mater. Sci., in press, doi:10.1016/ j.commatsci.2005.08.005.

[38] F. Drief, M. Tadjer, D. Mesri, H. Aourag, Catal. Today 89 (2004) 343.

[39] P. Dufek, P. Blaha, K. Schwarz, Phys. Rev. B 50 (1994) 7279.

[40] G.B. Bachelet, N.E. Christensen, Phys. Rev. B 31 (1985) 879.

[41] R. Dalven, Phys. Rev. Lett. 28 (1982) 91;

Phys. Rev. B 8 (1973) 6033;

Phys. Status Solidi B 62 (1974) K5. 